\title{
The Obesity Paradox in Cancer: a Review
}

\author{
Hannah Lennon $^{1,2}$ • Matthew Sperrin ${ }^{2}$ Ellena Badrick ${ }^{1,2}$ • Andrew G. Renehan ${ }^{1,2}$
}

Published online: 30 July 2016

(C) The Author(s) 2016. This article is published with open access at Springerlink.com

\begin{abstract}
There is a common perception that excess adiposity, commonly approximated by body mass index (BMI), is associated with reduced cancer survival. A number of studies have emerged challenging this by demonstrating that overweight and early obese states are associated with improved survival. This finding is termed the "obesity paradox" and is well recognized in the cardio-metabolic literature but less so in oncology. Here, we summarize the epidemiological findings related to the obesity paradox in cancer. Our review highlights that many observations of the obesity paradox in cancer reflect methodological mechanisms including the crudeness of BMI as an obesity measure, confounding, detection bias, reverse causality, and a specific form of the selection bias, known as collider bias. It is imperative for the oncologist to interpret the observation of the obesity paradox against the above methodological framework and avoid the misinterpretation that being obese might be "good" or "protective" for cancer patients.
\end{abstract}

Keywords Body mass index · Excess weight $\cdot$ Adiposity · BMI · Cancer · Prognosis · Cancer survival · Mortality · Overweight $\cdot$ Obesity $\cdot$ Epidemiology

This article is part of the Topical Collection on Integrative Care

Hannah Lennon

Hannah.lennon@manchester.ac.uk

1 Institute of Cancer Sciences, University of Manchester, Manchester, UK

2 Farr Institute, MRC Health eResearch Centre (HeRC North), University of Manchester, Manchester, UK

\section{Introduction}

Excess body adiposity is a major global public health problem, with $67 \%$ of the US, $63 \%$ of the UK, and $64 \%$ of Australia's population being classified as overweight or obese, by body mass index (BMI) criteria, in 2014 [1]. A report from the World Cancer Research Fund (WCRF) [2], and a systematic review with standardized meta-analysis from one of the present authors [3], established, approximately a decade ago, that elevated BMI is associated with increased cancer incidence for several common adult cancer types. There are now ten established obesity-related cancers listed by the WCRF, including post-menopausal breast, endometrial, ovarian, advanced prostate, colorectal, renal, pancreatic, liver, and gallbladder cancers and esophageal adenocarcinoma. There is a common perception that, compared with normal-weight patients, elevated BMI is also associated with poorer prognosis after cancer diagnosis. This certainly is observed in systematic reviews of the literature among women with breast cancer [4] and forms a key rationale for weight management recommendations among cancer survivors, endorsed by clinical guidelines, for example, by the American Society of Clinical Oncology [5], with similar recommendations from the American Cancer Society [6] and European Society for Medical Oncology [7].

However, a number of isolated historic studies [8-10] and an emerging number of recent studies [11-15] have observed that among patients with cancer, elevated BMI is associated with improved survival compared with normal-weight patients. The surprising nature of this finding suggests the existence of an "obesity paradox". This phenomenon is well described in the cardiovascular and metabolic literature [16-21] but less well appreciated in oncology. The repeated observation of the obesity paradox has spawned research that attempts to explain its occurrence. Posited explanations range from 
methodological (observed associations that contradict underlying causality due to confounding and bias) to clinical (seeking mechanistic explanations for obesity acting protectively in specific populations). In this review, we first explain what the obesity paradox is; summarize the current epidemiological findings for the association between overweight or obese status at cancer diagnosis and subsequent survival; review clinical and methodological explanations for the obesity paradox; and conclude with clinical implications and recommendations for further research.

\section{What Is the Obesity Paradox?}

A BMI of $22.5 \mathrm{~kg} / \mathrm{m}^{2}$ has been widely accepted as a midreference point for normal weight [22]. The obesity paradox occurs where the risk of outcome, typically mortality, is significantly reduced for BMI values above this referent, where an increased risk is expected. At very high BMI values, risk either returns to unity or is increased as illustrated in Fig. 1.

\section{Epidemiological Evidence}

There have been mixed findings in incident cancer populations where there has been exploration for the obesity paradox, with the paradox being observed in some studies [8-11, 13-15, 23], but not in all [24-26]. Consequently, there have been attempts to unify the conflicting results in the literature with systematic reviews on adiposity and cancer survival [27-30] but with inconsistent summaries.

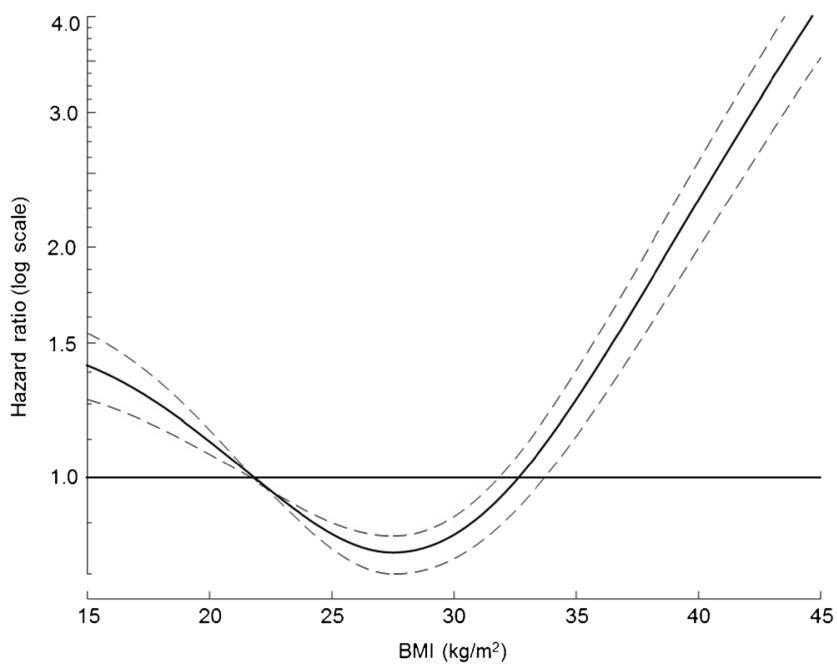

Fig. 1 An illustration of the obesity paradox. The vertical axis represents hazard ratio of mortality (log scale), compared with the baseline BMI of $22.5 \mathrm{~kg} / \mathrm{m}^{2}$. The plot represents a population in which the obesity paradox is observed, since the hazard ratio is below 1 in the overweight and obese range. The $95 \%$ confidence intervals are shown with dashed lines
The obesity paradox has been observed in different cancer settings including, for example, in patients with colorectal cancer undergoing surgery [11]; patients with renal cancer undergoing surgery $[10,12]$; patients with colorectal metastases undergoing liver resection [13]; elderly patients with acute myeloid leukemia [14]; and patients with lymphoma undergoing autologous hematopoietic cell transplantation [9]. The obesity paradox is not limited to non-metastatic disease and has been observed in a study of 4010 Taiwanese patients where the most common metastases were the lungs, liver, brain, and bone, requiring radiotherapy [15], and the hazard ratios decreased across BMI categories (overweight: HR 0.84 and obese: HR 0.67).

\section{General Points on Interpretation}

Given the variations in study findings, there is a need to have an initial framework to interpret whether the obesity paradox is a true or artificial association. There are two broad principles to consider in the study characteristics: (i) when (in relation to cancer diagnosis) BMI was determined and (ii) the age of the participants under study.

When BMI was determined is relevant. The recent WCRF report on the effect of risk factors on survival among women with breast cancer added a very useful classification - namely, determination of BMI either at pre-, peri-, or post-diagnosis (the later typically 12 months after the initial treatment) of cancer [31]. From these, different patterns of associations emerge. In a meta-analysis of 29 studies evaluating the impact of BMI on survival in patients with colorectal cancer, Wu et al. [30] observed that increasing pre-diagnosis BMI prognosticated for a poor survival but that post-treatment overweight was associated with improved survival, i.e., the obesity paradox. Table 1 demonstrates that the obesity paradox can be illustrated in all three settings of pre- [32], peri- [15], and postdiagnosis [11] for different cancer types.

Age is an additional attribute for consideration. For example, studies involving patients with leukemia are challenging to interpret due to the great age ranges of included individuals. Navarro and colleagues [33] showed that in over 4000 adults with acute myeloid leukemia under marrow transplantation, the obesity paradox was absent in young patients but present in those over age 60. Similar findings were noted by Brunner and colleagues, in a treatment cohort of adults with AML aged greater than 60 years [14].

\section{Explanations for the Obesity Paradox}

Determining whether the obesity paradox is a causal phenomenon among patients with cancer is clinically relevant, as it informs weight management strategies among cancer 


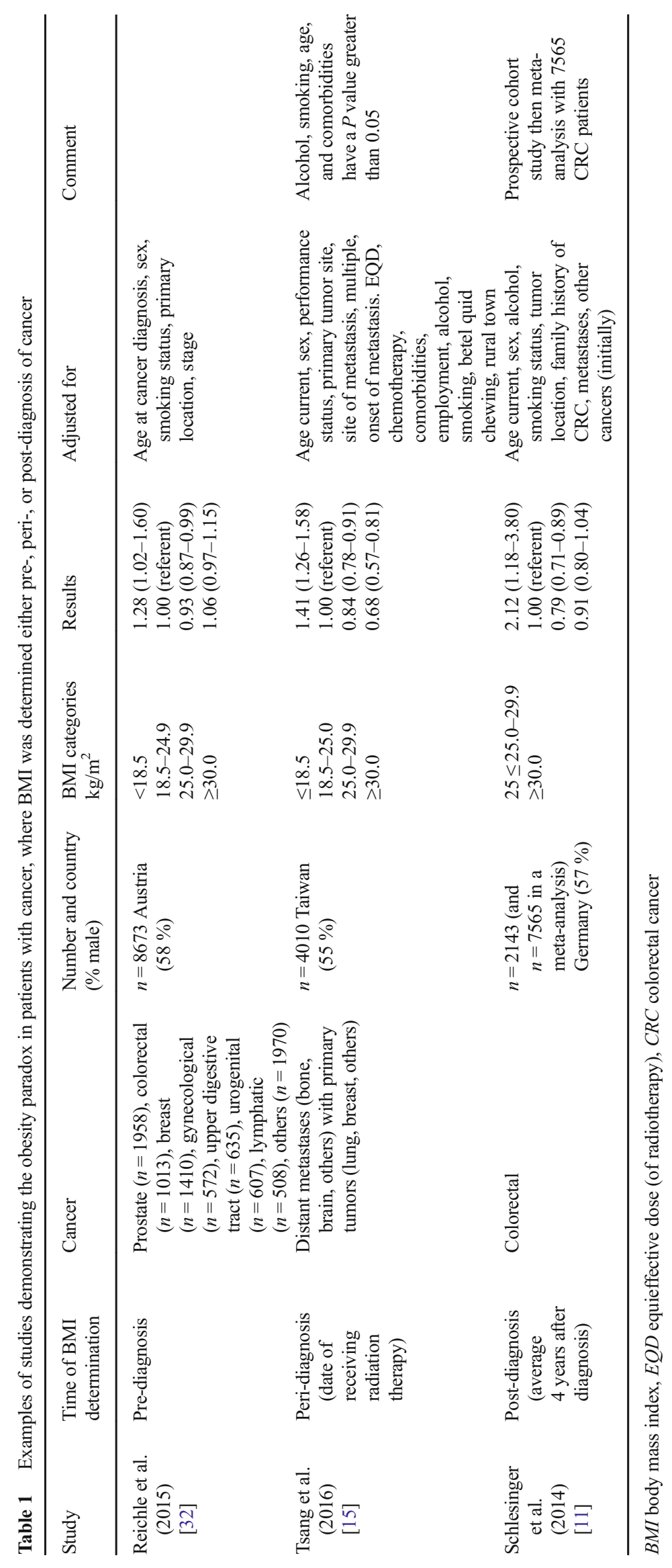


survivors. There are many potential causes of the obesity paradox, and understanding these is central to clinical implications. These are grouped into two broad categories [16]: the first is methodological and reflects spurious or artificial associations; the second is clinical and potentially reflects true associations and is clinically useful.

\section{Methodological Explanations}

\section{BMI as an Inadequate Measure of Adiposity}

BMI is commonly used as an approximation of general body adiposity in studies that have observed the obesity paradox. BMI is appealing as it is routinely measured in primary care and hospital settings and there are well-defined criteria for normal, overweight, and obese categories. However, BMI is a relatively crude measure of body adiposity and body composition and does not differentiate between lean mass and fat mass. In turn, body composition varies with age, sex, and ethnicity [22], such that there are currently no specific agegender-ethnicity indices to define obesity in a standardized manner. Thus, for example, in a cancer population, overweight individuals (defined by BMI) might be younger with high muscle mass (compared with normal weight), explaining their better outcome compared with normal weight.

The paradox might not exist if alternate measures of body composition or adipose tissue were used. Thus, for example, we found no examples of studies in patients with cancer demonstrating the obesity paradox when anthropometric measures other than BMI or body composition indices were used. Alternate indices include measurements such as waist circumference, waist to hip ratio, skinfold, and body composition assessment techniques such as dual-energy X-ray absorptiometry, CT, and MRI, and quantify different body fat components such as subcutaneous adipose tissue (SAT) and visceral adipose tissue (VAT) [34, 35]. Gonzalez and colleagues [36] recently explored this hypothesis and showed that the obesity paradox was present in 175 patients with various cancers (breast, gynecological, head and neck, lung, and gastrointestinal) when BMI was the exposure of interest but disappeared when obesity was defined using fat mass index and fat-free mass index.

\section{Confounding}

Confounding occurs when there are variables that are associated with both the outcome (death) and the exposure (obesity) and are not on the causal pathway between them. A common example is smoking, where BMI values are generally lower in current smokers than in never smokers. Other examples include deprivation, socioeconomic status, physical activity, and diet. It is difficult to adjust for all confounding factors, as many are unobserved. Measurement error may lead to incomplete removal of confounding, and to avoid this in the smoking context, some studies exclude smokers from their analysis [37], but usually, this is at the cost of disregarding a large proportion of the sample. Another approach would be to quantify exposure more accurately, for example, in terms of smoking duration and intensity using pack-years variables or cumulative lifetime exposure.

An example of confounding as a source of a spurious obesity paradox is illustrated in clinical treatment series reported by Hakimi and colleagues [12] in 2119 patients with renal cell carcinoma undergoing surgical resection at Memorial Sloan Kettering Cancer Center. Higher BMI was associated with reduced cancer-specific mortality in univariable analyses $(P<0.005)$, but this association was lost after adjusting for stage and grade $(P>0.10)$.

\section{Selection Bias/Collider Stratification Bias}

The obesity paradox might be due to a specific form of selection bias, known as the collider stratification bias, caused during the statistical analysis due to conditioning on a subpopulation selected based on a collider variable. In turn, a collider variable is one with at least two causes common to the risk of the variable and the outcome of interest. For example, cancer incidence is a collider variable because it is "caused" by both obesity and other risk factors (e.g., smoking). There is a wellrecognized inverse relationship between BMI and smoking. Thus, cancer patients who are not obese are more likely to have other risk factors, such as smoking, and in the analyzed subpopulation, an inverse association is artificially generated (or strengthened) between obesity and the other risk factors. Additionally, Banack and Kaufman [38・• d demonstrate how confounding due to smoking is increased in the presence of collider stratification bias. In a contrary direction, Sperrin et al. [39••], using an equation-derived approach within a counterfactual framework, show that the biases attributable to collider stratification are small and cannot explain the large paradoxical relationships seen in epidemiological studies.

\section{Detection Bias}

A further dimension is detection bias. This is the cooccurrence of two diagnoses together. Thus, for example, being overweight and obese is associated with the development of diabetes and cardiovascular disease. Where patients present with new diagnoses of these conditions, they undergo several investigations, which in turn detect incidental diseases including silent cancers - a form of "opportunistic surveillance". This overestimation of the occurrence of cancer diagnosis concurrent with a new diagnosis of diabetes is well recognized [40]. These silent cancers might have a low-stage disease with generally good prognosis and account for the obesity paradox in overweight and obese patients. One approach to minimize 
detection bias is to adjust for tumor stage at presentation or alternatively where the date of diagnosis of, say, diabetes is known, use a washout of 2 years to "bypass" the detection bias.

\section{Reverse Causality}

Reverse causality refers to the phenomenon that some normalweight patients may have previously been obese but lost weight due to illness, here cancer. Cancer is known to cause weight loss by loss of appetite or increased metabolic demands. The extent of weight loss correlates with initial BMI and occurs in patients with early-stage as well as late-stage tumors [41]. The potential causal link of reverse causality with the obesity paradox has been shown in several examples. Thus, Gelber and colleagues [42] evaluated the relationship between BMI and mortality in 99,253 male physicians in the Physicians' Health Study and initially showed a U-shaped association with all-cause mortality, which converted to a linear relationship in their optimal model excluding men who died within 2 years of initial assessment. Similarly, Tseng [43] evaluated a nationally representative cohort of 89,056 Taiwanese patients with type 2 diabetes, matched with the National Death Certificate Database, and on initial analysis found that BMI was inversely associated with mortality from all-cause, cancer, and diabetes complications, but after excluding patients with a follow-up duration less than 2 years, BMI categories were not significantly prognostic for cancer-related mortality, suggesting a bias induced by cancer-induced weight loss.

Reverse causality is better explored and minimized by using longitudinal data to obtain a description of the patient's usual weight and its trajectory up to cancer diagnosis. Weight histories (repeated weight measurements) give a useful dimension of obesity exposure, but there is a paucity of such data. Research to define obesity-equivalence of pack-years will be informative. A simpler approach when weight histories are not available is to include an individual's maximum lifetime BMI and is robust to confounding by illness-induced weight loss. Stokes and Preston [37] have illustrated this approach demonstrating a reduction in biases when including maximum lifetime BMI into their models. This approach is similar to evaluating weight change, for example, the rate of change and size of variability over time [28, 44].

\section{Clinical Explanations}

\section{Tumor Biology Is Less Aggressive}

There are some examples where tumors among obese patients have less aggressive characteristics compared with those among normal-weight patients. In obese women with endometrial cancer, there is a predominance of good prognosis type 1 tumors compared with poor prognosis type 2 endometrial cancer. Tumors are molecularly heterogeneous, and it is speculated that obesity is associated with less aggressive biological subtypes. For renal carcinoma, obesity is associated with more indolent molecular variants (for example, reduced fatty acid synthase, FASN, gene expression) [12], while in contrast, for ovarian cancer, elevated BMI is associated with good prognosis cancers (low-grade serous and endometrioid), but within this histological type subpopulation, there is a linear positive association between BMI and mortality, which is absent in high-grade serous ovarian cancers [45].

\section{Tumors Respond Better in Obese Patients}

The overweight and obese state might influence treatment outcomes, both in terms of how the tumor (changed for consistency) behaves to treatment and in terms of the differential pharmacokinetics of cancer treatment regimens. For example, high-intra-abdominal fat volume predicts for greater doxorubicin exposure and hematologic toxicities in women with breast cancer compared with body surface area [46]. Similarly, overweight and obese patients might be differentially allocated to less radical cancer surgery, though Gurunathan and Myles [47] point out the limitations of BMI as a predictor of peri-operative complication risk and indicate that mildly obese and overweight patients outperform normal-weight patients after many types of surgeries.

A specific mention is worthwhile for the complex interrelationships between adjuvant chemotherapy (after curative resection) and the overweight/obese state. There is a wellrecognized clinical practice among many oncologists to dose cap chemotherapy in obese patients with body surface area (BSA) greater than 2.0, and together with differential allocation and differential adherence to adjuvant chemotherapy, obese patients may simply have poorer outcomes compared with normal-weight patients because they are sub-optimally treated. This is illustrated by Sinicrope and colleagues [48], who examined the prognostic impact of BMI in 25,291 patients with stage II and III colon carcinoma within the Adjuvant Colon Cancer Endpoints (ACCENT) database, a consortium of randomized trials of 5-fluorouracil-based adjuvant chemotherapy. With disease-free survival (DFS) as a key outcome measure, compared with normal-weight patients, they showed a significant reduction in DFS limited to men with class 2 and 3 obesity (BMI $\geq 35.0 \mathrm{~kg} / \mathrm{m}^{2}$ ) but an improved survival for overweight and class 1 obese (BMI 25.0 to $34.9 \mathrm{~kg} / \mathrm{m}^{2}$ ) men, i.e., the obesity paradox. There were different dose-capping practices among the trials, and there was lack of data on chemotherapy adherence by BMI status, such that the study was unable to conclude whether or not these confounders contributed to the differential impact of BMI states on survival. 


\section{Energy Reserve or Hibernation Hypothesis}

A third hypothesis is that excess adipose tissue serves as a nutrient reserve and confers a survival advantage in times of stress, such as anti-cancer treatment. This is akin to the hibernation theories in evolutional biology whereby species store up energy in anticipation of harsh times ahead. On a parallel note, it remains unclear if obesity drives cancer progression, whether it is due to excess adiposity or the energy imbalance [49].

\section{Conclusions and Future Directions}

This review has highlighted the mixed findings in studies evaluating the obesity paradox in cancer populations. In terms of interpreting these studies, and designing future studies on this topic, there is a need to apply a methodological framework to determine whether the obesity paradox is a true or spurious relationship for a given setting. If a framework is not used, mistaken interpretations can be reached. Thus, in the cardiovascular literature, some commentators have concluded that the obesity paradox is a true causal association arguing that the optimum body weight is above the normal BMI range in individuals with some chronic diseases [17].

First, where the primary interest is the effect of obesity on survival, it is preferred to incorporate as much information regarding the patients' weight history, i.e., consider the patient's BMI trajectory throughout a long period of time or ideally through the whole life-course. Variations on this include modeling BMI at pre-, peri-, and post-diagnosis of cancer. An alternative is to use maximum lifetime weight and weight variability measures.

Second, it is important to work within datasets with richness for potential confounding. The following are potential effect modifiers or confounders of the relationship between BMI and survival but are not always captured: smoking, hormonal replacement therapy, and ethnicity. Cancers diagnosed through screening programs have better prognosis than nonscreened cancer, and in turn, obesity tends to be associated with lower uptake rates in cancer screening.

Third, conflicting findings may be partly explained by heterogeneity within cancer types, the timing of when BMI was determined, unmeasured confounders, and statistical biases. To further understand the observed associations, directions for future research include (i) improving the "subtyping" of cancer by better recording of staging, tumor type; (ii) improving data linkage so BMI, adiposity measures, and confounding variables can readily be extracted from records and incorporated; and (iii) further research into the gender-specific links of the effect of obesity and overweight on survival in cancer populations.
It is imperative for the oncologist to interpret the observation of the obesity paradox against the above methodological framework and avoid the misinterpretation that being obese might be "good" or "protective" for cancer patients. "First, do no harm'.

This study was partly supported by the University of Manchester's Health eResearch Centre (HeRC) funded by the Medical Research Council (MRC) Grant MR/K006665/1 and the National Awareness and Early Detection Initiative funding scheme from Cancer Research UK Grant A17962.

\section{Compliance with Ethical Standards}

Conflict of Interest Hannah Lennon, Matthew Sperrin, Ellena Badrick, and Andrew G. Renehan declare that they have no conflict of interest.

Human and Animal Rights and Informed Consent This article does not contain any studies with human or animal subjects performed by any of the authors.

Open Access This article is distributed under the terms of the Creative Commons Attribution 4.0 International License (http:// creativecommons.org/licenses/by/4.0/), which permits unrestricted use, distribution, and reproduction in any medium, provided you give appropriate credit to the original author(s) and the source, provide a link to the Creative Commons license, and indicate if changes were made.

\section{References}

Papers of particular interest, published recently, have been highlighted as:

•. Of major importance

1. WHO. GHO|By category $\mid$ Overweight (body mass index $>=25$ ) (age-standardized estimate) — data by country [Accessed 18 March 2016].

2. WCRF A. Food, nutrition, physical activity, and the prevention of cancer: a global perspective. World Cancer Research Fund and American Institute For Cancer Research. 2nd ed. Washington: American Institute for Cancer Research; 2007.

3. Renehan AG, Tyson M, Egger M, Heller RF, Zwahlen M. Bodymass index and incidence of cancer: a systematic review and metaanalysis of prospective observational studies. Lancet. 2008;371: 569-78

4. Chan DS, Vieira AR, Aune D, Bandera EV, Greenwood DC, McTiernan A, et al. Body mass index and survival in women with breast cancer-systematic literature review and meta-analysis of 82 follow-up studies. Ann Oncol. 2014;25(10):1901-14.

5. Ligibel JA, Alfano CM, Courneya KS, Demark-Wahnefried W, Burger RA, Chlebowski RT, et al. American Society of Clinical Oncology position statement on obesity and cancer. J Clin Oncol. 2014;32(31):3568-74.

6. Rock CL, Doyle C, Demark-Wahnefried W, Meyerhardt J, Courneya KS, Schwartz AL, et al. Nutrition and physical activity guidelines for cancer survivors. CA Cancer J Clin. 2012;62(4): 243-74.

7. Senkus E, Kyriakides S, Penault-Llorca F, Poortmans P, Thompson A, Zackrisson S, et al. Primary breast cancer: ESMO Clinical 
Practice Guidelines for diagnosis, treatment and follow-up. Ann Oncol. 2013;24 Suppl 6:vi7-vi23.

8. Hines RB, Shanmugam C, Waterbor JW, McGwin G, Funkhouser E, Coffey CS, et al. Effect of comorbidity and body mass index on the survival of African-American and Caucasian patients with colon cancer. Cancer. 2009;115:5798-806.

9. Navarro WH, Loberiza Jr FR, Bajorunaite R, van Besien K, Vose JM, Lazarus HM, et al. Effect of body mass index on mortality of patients with lymphoma undergoing autologous hematopoietic cell transplantation. Biol Blood Marrow Transplant. 2006;12:541-51.

10. Parker AS, Lohse CM, Cheville JC, Thiel DD, Leibovich BC, Blute ML. Greater body mass index is associated with better pathologic features and improved outcome among patients treated surgically for clear cell renal cell carcinoma. Urology. 2006;68:741-6.

11. Schlesinger S, Siegert S, Koch M, Walter J, Heits N, Hinz S, et al. Postdiagnosis body mass index and risk of mortality in colorectal cancer survivors: a prospective study and meta-analysis. Cancer Causes Control. 2014;25:1407-18.

12. Hakimi AA, Furberg H, Zabor EC, Jacobsen A, Schultz N, Ciriello $\mathrm{G}$, et al. An epidemiologic and genomic investigation into the obesity paradox in renal cell carcinoma. J Natl Cancer Inst. 2013;105: 1862-70.

13. Amptoulach S, Gross G, Kalaitzakis E. Differential impact of obesity and diabetes mellitus on survival after liver resection for colorectal cancer metastases. J Surg Res. 2015;199:378-85.

14. Brunner AM, Sadrzadeh H, Feng Y, Drapkin BJ, Ballen KK, Attar $\mathrm{EC}$, et al. Association between baseline body mass index (BMI) and overall survival among patients over age 60 with acute myeloid leukemia (AML). Am J Hematol. 2013;88:642-6.

15. Tsang NM, Pai PC, Chuang CC, Chuang WC, Tseng CK, Chang $\mathrm{KP}$, et al. Overweight and obesity predict better overall survival rates in cancer patients with distant metastases. Cancer Med 2016: n/a-n/a.

16. Banack HR, Kaufman JS. The obesity paradox: understanding the effect of obesity on mortality among individuals with cardiovascular disease. Prev Med. 2014;62:96-102.

17. Doehner W, Haehling SV, Anker SD. Protective overweight in cardiovascular disease: moving from 'paradox' to 'paradigm'. Eur Heart J 2015:ehv414.

18. Lavie CJ, Milani RV, Ventura HO. Obesity and cardiovascular disease: risk factor, paradox, and impact of weight loss. J Am Coll Cardiol. 2009;53:1925-32.

19. McAuley PA, Artero EG, Sui X, Lee D-c, Church TS, Lavie CJ, et al. The obesity paradox, cardiorespiratory fitness, and coronary heart disease. Mayo Clin Proc. 2012;87:443-51.

20. McAuley PA, Blair SN. Obesity paradoxes. J Sports Sci. 2011;29: 773-82.

21. Tobias DK, Pan A, Jackson CL, O'Reilly EJ, Ding EL, Willett WC, et al. Body-mass index and mortality among adults with incident type 2 diabetes. N Engl J Med. 2014;370(3):233-44.

22. Arnold M, Leitzmann M, Freisling H, Bray F, Romieu I, Renehan A, et al. Obesity and cancer: an update of the global impact. Cancer Epidemiol. 2016;41:8-15.

23. Daniel CR, Shu X, Ye Y, Gu J, Raju GS, Kopetz S, et al. Severe obesity prior to diagnosis limits survival in colorectal cancer patients evaluated at a large cancer centre. Br J Cancer. 2016;114: 103-9.

24. Crosbie EJ, Roberts C, Qian W, Swart AM, Kitchener HC, Renehan AG. Body mass index does not influence post-treatment survival in early stage endometrial cancer: results from the MRC ASTEC trial. Eur J Cancer. 2012;48:853-64.

25. Yoon HH, Lewis MA, Shi Q, Khan M, Cassivi SD, Diasio RB, et al. Prognostic impact of body mass index stratified by smoking status in patients with esophageal adenocarcinoma. J Clin Oncol. 2011;29:4561-7.
26. Zhang S, Folsom AR, Sellers TA, Kushi LH, Potter JD. Better breast cancer survival for postmenopausal women who are less overweight and eat less fat. The Iowa Women's Health Study. Cancer. 1995;76:275-83.

27. Feng Y-H. The association between obesity and gynecological cancer. Gynecol Minim Invasive Ther. 2015;4:102-5.

28. Parekh N, Chandran U, Bandera EV. Obesity in cancer survival. Annu Rev Nutr. 2012;32:311-42.

29. Parkin E, O'Reilly DA, Sherlock DJ, Manoharan P, Renehan AG. Excess adiposity and survival in patients with colorectal cancer: a systematic review. Obes Rev. 2014;15:434-51.

30. Wu S, Liu J, Wang X, Li M, Gan Y, Tang Y. Association of obesity and overweight with overall survival in colorectal cancer patients: a meta-analysis of 29 studies. Cancer Causes Control. 2014;25:1489 502.

31. WCRF. World Cancer Research Fund International. Continuous Update Project Report: diet, nutrition, physical activity, and breast cancer survivors. 2014. Available at:www.wcrf. org/sites/default/files/Breast-Cancer-Survivors-2014-Report.pdf [Accessed 20th Dec 2014]. 2014.

32. Reichle K, Peter RS, Concin H, Nagel G. Associations of prediagnostic body mass index with overall and cancer-specific mortality in a large Austrian cohort. Cancer Causes Control. 2015;26: $1643-52$.

33. Navarro WH, Agovi M-A, Logan BR, Ballen K, Bolwell BJ, Frangoul $\mathrm{H}$, et al. Obesity does not preclude safe and effective myeloablative hematopoietic cell transplantation (HCT) for acute myelogenous leukemia (AML) in adults. Biol Blood Marrow Transplant. 2010;16:1442-50.

34. Coe PO, O'Reilly DA, Renehan AG. Excess adiposity and gastrointestinal cancer. Br J Surg. 2014;101(12):1518-31. discussion 1531.

35. Shuster A, Patlas M, Pinthus JH, Mourtzakis M. The clinical importance of visceral adiposity: a critical review of methods for visceral adipose tissue analysis. Br J Radiol. 2012;85(1009):1-10.

36. Gonzalez MC, Pastore CA, Orlandi SP, Heymsfield SB. Obesity paradox in cancer: new insights provided by body composition. Am J Clin Nutr. 2014;99(5):999-1005.

37. Stokes A, Preston SH. Revealing the burden of obesity using weight histories. Proc Natl Acad Sci U S A. 2016;113:572-7.

38.• Banack HR, Kaufman JS. From bad to worse: collider stratification amplifies confounding bias in the "obesity paradox". Eur J Epidemiol. 2015;30(10):1111-4. A prospective cohort study of 15,792 US men and women showing that confounding bias increases in the presence of collider stratification bias.

39.• Sperrin M, Candlish J, Badrick E, Renehan A, Buchan I. Collider bias cannot explain most of the obesity paradox [in press]. Epidemiology 2016. A simulation study that collider bias is an infrequent cause of the obesity paradox.

40. Johnson JA, Bowker SL, Richardson K, Marra CA. Time-varying incidence of cancer after the onset of type 2 diabetes: evidence of potential detection bias. Diabetologia. 2011;54:2263-71.

41. Renehan AG, Crosbie EJ, Campbell PT. Re: Prediagnosis body mass index, physical activity, and mortality in endometrial cancer patients. J Natl Cancer Inst. 2014;106(2):djt375.

42. Gelber RP, Kurth T, Manson JE, Buring JE, Gaziano JM. Body mass index and mortality in men: evaluating the shape of the association. Int J Obes. 2007;31:1240-7. 200.

43. Tseng C-H. Obesity paradox: differential effects on cancer and noncancer mortality in patients with type 2 diabetes mellitus. Atherosclerosis. 2013;226:186-92.

44. Renehan AG, Flood A, Adams KF, Olden M, Hollenbeck AR, Cross AJ, et al. Body mass index at different adult ages, weight change, and colorectal cancer risk in the National Institutes of Health-AARP Cohort. Am J Epidemiol. 2012;176:1130-40. 
45. Nagle CM, Dixon SC, Jensen A, Kjaer SK, Modugno F, DeFazio A, et al. Obesity and survival among women with ovarian cancer: results from the Ovarian Cancer Association Consortium. Br J Cancer. 2015;113:817-26.

46. Wong AL, Seng KY, Ong EM, Wang LZ, Oscar H, Cordero MT, et al. Body fat composition impacts the hematologic toxicities and pharmacokinetics of doxorubicin in Asian breast cancer patients. Breast Cancer Res Treat. 2014;144:143-52.

47. Gurunathan U, Myles PS. Limitations of body mass index as an obesity measure of perioperative risk. Br J Anaesth. 2016;116:319-21.
48. Sinicrope FA, Foster NR, Yothers G, Benson A, Seitz JF, Labianca $\mathrm{R}$, et al. Body mass index at diagnosis and survival among colon cancer patients enrolled in clinical trials of adjuvant chemotherapy. Cancer. 2013;119(8):1528-36.

49. Demark-Wahnefried W, Platz EA, Ligibel JA, Blair CK, Courneya KS, Meyerhardt JA, et al. The role of obesity in cancer survival and recurrence. Cancer Epidemiol Biomark Prev. 2012;21:1244-59. 